\title{
Giant Pedunculated Hepatic Hemangioma: A Case Report and Literature Review
}

\author{
Abdallah Al Farai ${ }^{a} \quad$ Lénaïg Mescam $^{b}$ Valeria De Luca ${ }^{c}$ \\ Audrey Monneur ${ }^{d} \quad$ Delphine Perrot $^{d} \quad$ Jerome Guiramand $^{a}$ \\ Jean-Robert Delpero ${ }^{\mathrm{a}}$ e François Bertuccid, e \\ aDepartment of Surgical Oncology, Institut Paoli-Calmettes, Marseille, France; \\ ${ }^{b}$ Department of Pathology, Institut Paoli-Calmettes, Marseille, France; 'Department of \\ Radiology, Institut Paoli-Calmettes, Marseille, France; dDepartment of Medical Oncology, \\ Institut Paoli-Calmettes, Marseille, France; eFaculty of Medicine, Aix-Marseille University, \\ Marseille, France, Marseille, France
}

\section{Keywords}

Giant pedunculated hemangioma $\cdot$ Liver $\cdot$ Surgery

\section{Abstract}

Hepatic hemangioma is the most common benign hepatic tumor, and most of them are small in size and asymptomatic. Giant hepatic hemangiomas are uncommon, but pedunculated giant hemangiomas are even rarer and often difficult to diagnose because of their exophytic development. We report here on a 48-year-old man with a symptomatic pedunculated giant hepatic hemangioma and hepatic angiomatosis, mimicking a gastric gastrointestinal stromal tumor with liver metastases. The preoperative diagnosis was suspected on imaging including CT scan and MRI. The patient was successfully operated (left hepatic lobectomy), without any compli- 


\section{Case Reports in Oncology}

Al Farai et al:: Giant Pedunculated Hemangioma of the Liver

cation, and the pathological analysis confirmed the diagnosis. We reviewed the English literature, and to our knowledge, our case represents the largest case reported so far when compared with the 19 other informative cases.

(C) 2018 The Author(s)

Published by S. Karger AG, Basel

\section{Introduction}

Hepatic hemangiomas are the most common benign hepatic lesions. The prevalence of these congenital vascular malformations, between 3 and $20 \%[1,2]$, is probably underestimated. Hepatic hemangiomas are more frequent in women because of the influence of female sex hormones on their growth. They can be found at any age, but $60-80 \%$ are diagnosed in 30 - to 50 -year-old patients. Most frequently, the lesion is unique $(75 \%$ of the cases) and of variable size; and the right lobe is the most common site. In the majority of patients, the lesion is asymptomatic and requires no treatment, the diagnosis being done incidentally by imaging modalities such as ultrasonography (US), computed tomography (CT) scan, or magnetic resonance imaging (MRI). Hepatic hemangiomas larger than $4 \mathrm{~cm}$ are rare and have been classified as giant hemangioma, although such definition has been controversial (cutoffs between 4 and $10 \mathrm{~cm}$ ) during the last few years [2].

Pedunculated giant hemangiomas are even rarer and often difficult to diagnose because of their exophytic development. We report here on a 48-year-old man with symptomatic pedunculated giant hepatic hemangioma and hepatic angiomatosis, mimicking a gastric gastrointestinal stromal tumor with liver metastases. The preoperative diagnosis was based on imaging. The patient was successfully operated on, without any complication. To our knowledge, our case represents the largest case reported so far in the English literature when compared with the 19 other informative cases [3-10].

\section{Case Presentation}

On June 2016, a Caucasian 48-year-old man consulted his general practitioner for diarrhea, vomiting and epigastric pain, which increased while coughing or leaning forward. He had had a blunt abdominal trauma at work 1 month prior to his presentation. The clinical examination revealed a huge palpable abdominal mass occupying most of the epigastric and right hypochondriac region. The blood tests were almost normal except of a mild increase in gamma-glutamyl transferase. The abdominal US showed a voluminous $(18 \times 10 \mathrm{~cm})$ heterogeneous mass located in the epigastric region in front of the pancreas and pushing the left liver lobe anteriorly and laterally. There were also multiple (5-6) intrahepatic lesions evoking hepatic hemangiomas. The abdominopelvic contrast-enhanced CT scan showed a voluminous prepancreatic tissue tumor with heterogeneous predominantly peripheral enhancement, measuring about $20 \times 9 \times 16 \mathrm{~cm}$ (Fig. 1a, b), associated with multiple hypodense hepatic nodules evoking hemangiomas, although two of them might also evoke metastatic lesions. The patient was referred to our institution for a suspected diagnosis of gastric submucosal tumor with likely hepatic metastases. The thoracic contrast-enhanced CT scan was normal. 


\section{Case Reports in Oncology}

He had no specific familial and medical personal history, except allergic asthma and active smoking. His performance status was zero. The digestive symptoms persisted and clinical examination confirmed this voluminous palpable abdominal mass. The case was discussed in the Sarcoma Multidisciplinary Board, and the diagnosis of giant pedunculated liver hemangioma was suspected. It was decided to perform a liver MRI which showed several typical hemangiomas disseminated throughout the liver, with classical enhancement after contrast injection. The largest one was a pedunculated hemangioma originating from the third hepatic segment, with exophytic development, descending in the epi- and mesogastric region, pushing the pancreas backwards. Its dimensions were $20 \times 8 \times 14 \mathrm{~cm}$ (Fig. 1c, d). There was no radiological argument in favor of eventual posttraumatic rupture, torsion or hemorrhage. Due to this typical aspect of hepatic angiomatosis with symptomatic giant pedunculated hemangioma and the risk of torsion and rupture, the surgical excision was decided to be performed without any preoperative biopsy.

The patient was operated on in July 2016. Through a large median laparotomy incision, a left hepatic lobectomy was done (Fig. 2) without any intraoperative incident; the blood loss was estimated to be at around $150 \mathrm{~mL}$. The postoperative period was uneventful and the patient was discharged home at the fourth postoperative day. The pathological examination of operative specimen (Fig. 3) revealed a giant hemangioma, with multiple associated infarcted or hemorrhagic vascular lesions. The hemangioma was formed by multiple dilated thin-walled vascular channels, lined by regular endothelial cells, without any atypia. Immunohistochemistry confirmed the diagnosis of hemangioma, with positive ERG staining of endothelial cells. The surrounding hepatic parenchyma showed multiple millimetric hemangiomas, characterized by congestive ectatic vessels. In May 2018, 23 months after surgery, the patient is alive without any relapse on liver MRI.

\section{Discussion}

Giant pedunculated hepatic hemangiomas are very rare benign tumors, with only 24 cases previously reported [3-10] in the English literature. We report here the largest lesion $(20 \mathrm{~cm})$ reported so far. Nineteen out of these 24 cases are reported with precision in the literature and their characteristics and those of our case are summarized in Table 1.

There were more women $(n=14)$ than men $(n=6)$ observed with classical hepatic hemangiomas. The median age at diagnosis was 53 years (range, 28-71). The most frequent location was the left hepatic lobe, by contrast with that of non-giant pedunculated hemangiomas. The median tumor size was $11.5 \mathrm{~cm}$, ranging from 4 to $20 \mathrm{~cm}$. In 6 out of 20 cases (30\%), the disease was asymptomatic and diagnosed incidentally, on imaging studies performed as routine examinations or for other reasons than the evaluation of a possible liver mass. By contrast, the disease was symptomatic in 14 cases (70\%). This larger rate of symptomatic disease at diagnosis, when compared with classical hepatic hemangiomas, is due to the larger size, the extrahepatic development and perhaps the most frequent left location, which may determine compression of the intra- and extrahepatic biliary ducts, vascular structures or adjacent organs such as the digestive tube. Other potential complications include inflammation, spontaneous or traumatic or iatrogenic rupture, intratumoral bleeding, Kasabach-Merritt syndrome 


\section{Case Reports in Oncology}

(consumptive coagulopathy associating hemolytic anemia and microangiopathy, thrombocytopenia, fibrinolysis, and hypofibrinogenemia), and congestive heart failure resulting from arteriovenous shunt. There is no evidence for malignant transformation of hemangiomas in the literature, and hemangioma and hemangiosarcoma have different developmental pathways and derive from a different cellular clone [11]. Classical complications of giant pedunculated forms include torsion due to a long and mobile pedicle, and infarction. Among the 14 symptomatic cases reported, 4 showed acute complications linked to torsion $(n=2)$, digestive obstruction $(n=1)$, and inflammation $(n=1)$. The 10 non-acute symptomatic cases presented 1 or several symptoms (abdominal pain, discomfort, dyspepsia, nausea, vomiting, diarrhea, icterus, abdominal palpable mass), among which the most represented was abdominal pain, which is not specific. In hepatic hemangioma, the pain may be caused by intratumoral thrombosis, tumor inflammation, tumor hemorrhage or even rupture. It is due to liver capsule distension because the tumor grows slowly, allowing the liver to adapt to this new anatomical situation. The pain can manifest after an abdominal trauma similar to what happened in our case.

The diagnosis of a giant pedunculated lesion may be difficult, despite the typical radiological presentation, because the thin pedicle may be undetectable on images, making it difficult to define the origin of the mass. The most used imaging modalities in diagnosis include US, CT scan and MRI, which remains the most sensitive and specific (>90\%) diagnostic method. Biopsy is not recommended because of the hemorrhagic risk. Three cases [7, 9] including our case mimicked submucosal gastric tumor such as gastrointestinal stromal tumor. Whereas the surgical treatment of hepatic hemangiomas is reserved for cases of giant or symptomatic lesions, uncertain diagnosis, lesions with complications, and rapidly growing lesion, it is systematically indicated for cases of pedunculated hemangiomas due to their tendency to torsion and rupture.

In conclusion, giant pedunculated hepatic hemangiomas are very rare benign tumors that should be included in the differential diagnosis of any mass located in the upper abdomen. Imaging, such as ultrasound, CT scan, and MRI, are used to diagnose these tumors. All giant pedunculated hemangiomas must be surgically removed.

\section{Acknowledgement}

Our work is supported by the Institut Paoli-Calmettes.

\section{Statement of Ethics}

We thank our patient, who kindly gave her consent for this publication.

\section{Disclosure Statement}

The authors declare no conflict of interest. 


\section{References}

1 Bajenaru N, Balaban V, Săvulescu F, Campeanu I, Patrascu T. Hepatic hemangioma - review. J Med Life. 2015;8(Spec Issue):4-11.

2 Di Carlo I, Koshy R, Al Mudares S, Ardiri A, Bertino G, Toro A. Giant cavernous liver hemangiomas: is it the time to change the size categories? Hepatobiliary Pancreat Dis Int. 2016 Feb;15(1):21-9.

3 Acharya M, Panagiotopoulos N, Bhaskaran P, Kyriakides C, Pai M, Habib N. Laparoscopic resection of a giant exophytic liver haemangioma with the laparoscopic Habib $4 \times$ radiofrequency device. World J Gastrointest Surg. 2012 Aug;4(8):199-202.

4 Candido PC, Pereira IM, Matos BA, Fontes MH, Pires TE, Costa PR. Giant pedunculated hemangioma of the liver. Radiol Bras. 2016 Jan-Feb;49(1):57-8.

5 Darzi A, Taheri H, Kamali Ahangar S, Mirzapour Shafiei A, Asghari Y. Torsion of a Giant Pedunculated Hemangioma of the Liver Presenting with Acute Abdomen: A Case Report. Iran Red Crescent Med J. 2016 Jul;18(8):e38198.

6 Ersoz F, Ozcan O, Toros AB, Culcu S, Bektas H, Sari S, et al. Torsion of a giant pedunculated liver hemangioma mimicking acute appendicitis: a case report. World J Emerg Surg. 2010 Jan;5(1):2.

7 Guenot C, Haller C, Rosso R. Giant pedunculated cavernous hepatic haemangioma: a case report and review of the literature. Gastroenterol Clin Biol. 2004 Aug-Sep;28(8-9):807-10.

8 Karatzas T, Smirnis A, Dimitroulis D, Patsouras D, Evaggelou K, Kykalos S, et al. Giant pedunculated hepatocellular carcinoma with hemangioma mimicking intestinal obstruction. BMC Gastroenterol. 2011 Sep;11(1):99.

9 Moon HK, Kim HS, Heo GM, Shin WG, Kim KH, Jang MK, et al. A case of pedunculated hepatic hemangioma mimicking submucosal tumor of the stomach. Korean J Hepatol. 2011 Mar;17(1):66-70.

10 Vivarelli M, Gazzotti F, D’Alessandro L, Pinna AD. Image of the month. Emergency presentation of a giant pedunculated liver haemangioma. Dig Liver Dis. 2010 Jun;42(6):456.

11 Liu L, Kakiuchi-Kiyota S, Arnold LL, Johansson SL, Wert D, Cohen SM. Pathogenesis of human hemangiosarcomas and hemangiomas. Hum Pathol. 2013 Oct;44(10):2302-11. 


\section{Case Reports in Oncology}

Case Rep Oncol 2018;11:476-484 www.karger.com/cro

Al Farai et al.: Giant Pedunculated Hemangioma of the Liver
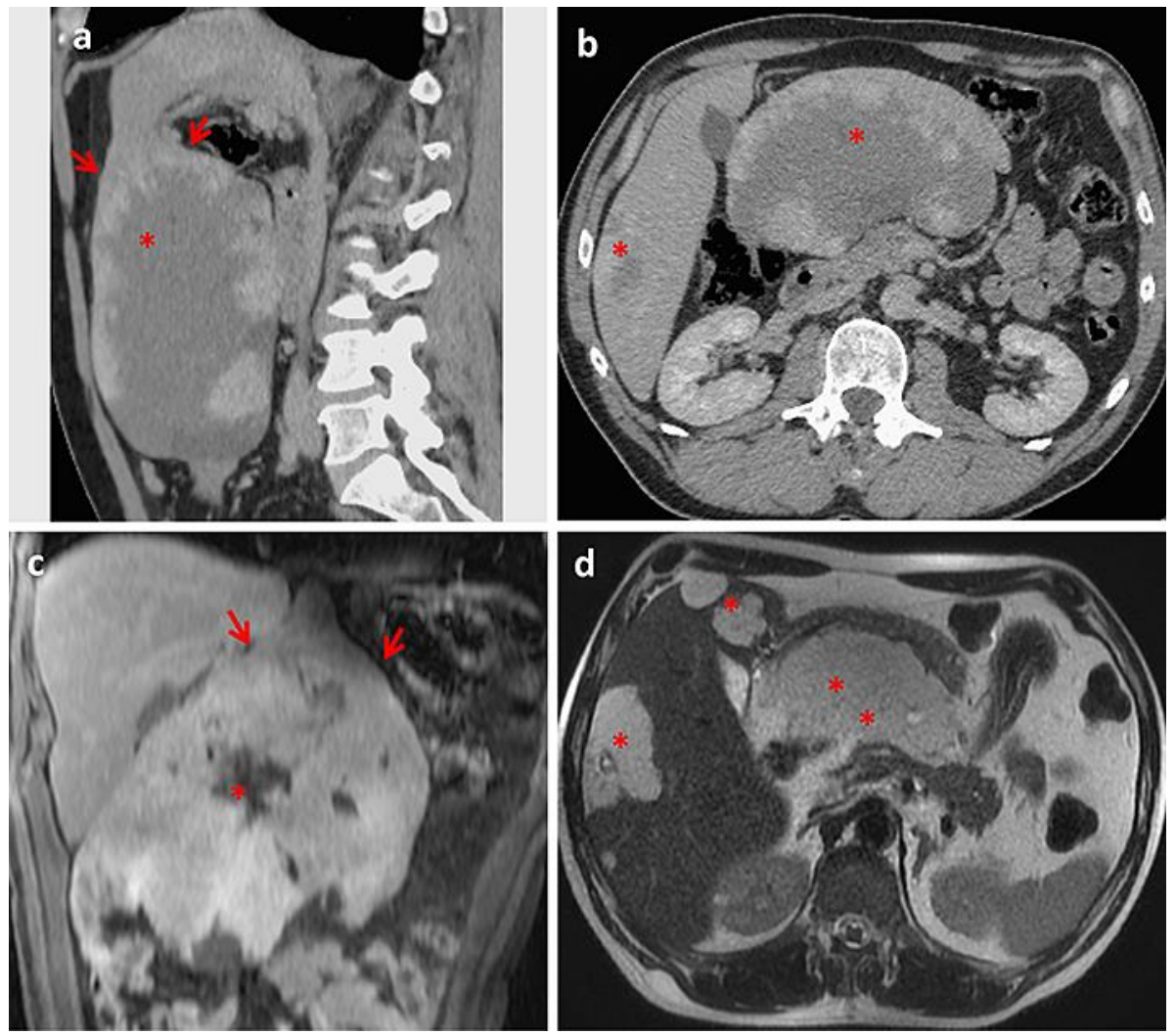

Fig. 1. Radiological aspects. a Sagittal CT scan at the portal venous phase showing a prepancreatic and subhepatic, large, solid, and well-defined mass (red star) with peripheral enhancement and the presence of the claw sign (arrows) suggests that this lesion arises from the third segment of the liver. The pedicle is also well visible. b Axial CT scan at the portal venous phase showing two hemangiomas (red stars) with progressive peripheral enhancement with more centripetal fill-in. c Coronal T1-weighted MRI showing the liver mass with the claw sign (red arrows] and progressive centripetal enhancement (inward) on delayed images (red star). d Axial T2-weighted MRI showing three liver hemangiomas (red stars) with signal hyperintense relative to liver parenchyma. 


\section{Case Reports in Oncology}
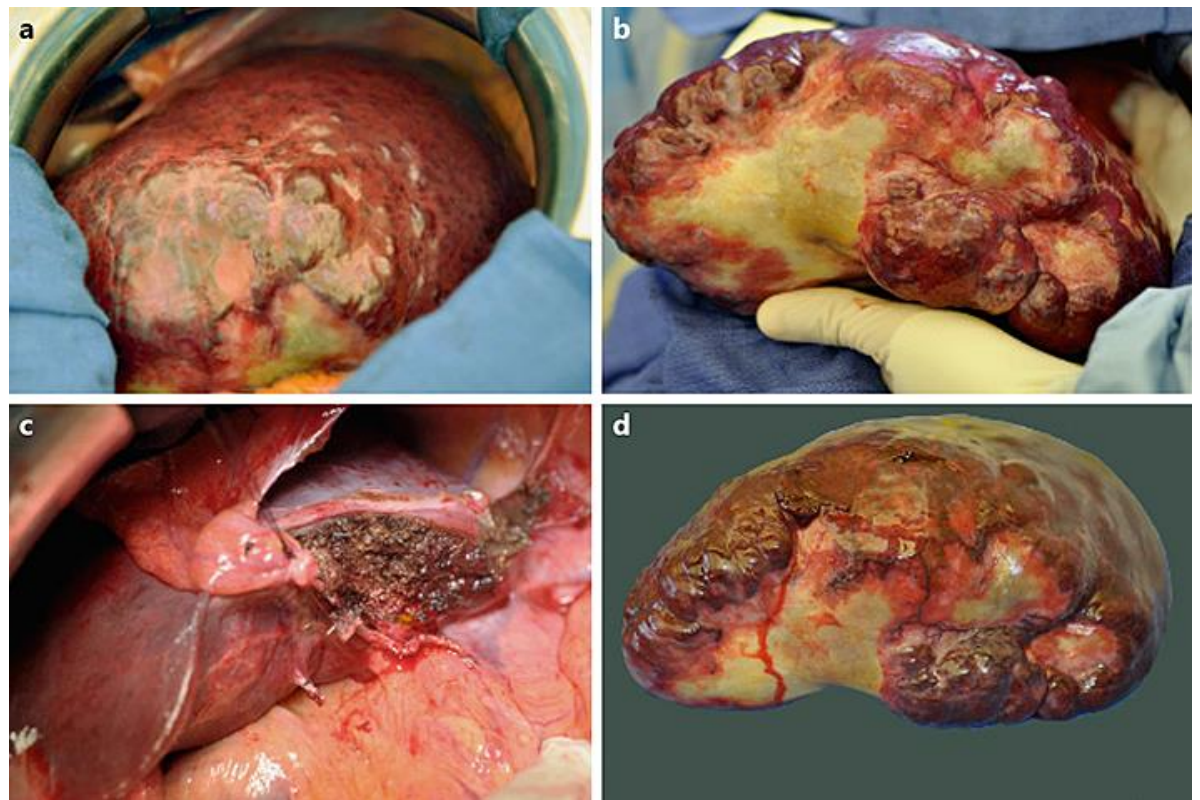

Fig. 2. Intraoperative aspects. a Anteroinferior side of the left hepatic lobe. The giant hemangioma occupies the left hepatic lobe and deforms the liver surface and gives an irregular multiloculated depressed white surface. $\mathbf{b}$ Posteroinferior side of the specimen. $\mathbf{c}$ Section surface on the remaining liver. The round ligament of the liver is suspended by the surgical suture. $\mathbf{d}$ Posteroinferior side of the specimen. 


\section{Case Reports in Oncology}

www.karger.com/cro

Al Farai et al.: Giant Pedunculated Hemangioma of the Liver
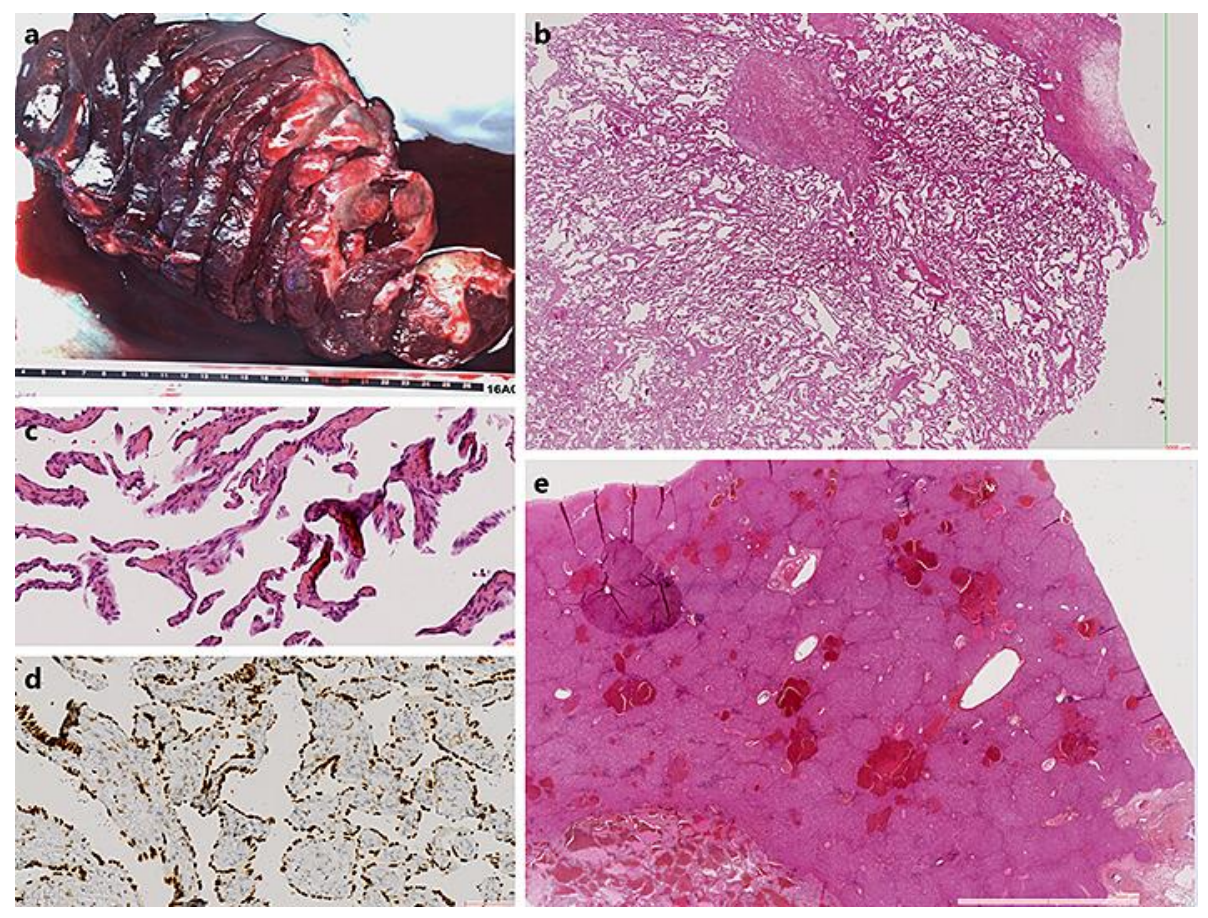

Fig. 3. Pathological aspects. a Left hepatectomy showing a giant hemangioma, with multiple associated infarcted or hemorrhagic vascular lesions. b Hemangioma is formed by multiple dilated thin-walled vascular channels (HES $\times 0.6$ ). c Vascular channels are lined by regular endothelial cells, with no atypia (HES $\times 10)$. $\mathbf{d}$ Endothelial cells express ERG after IHC staining $(E R G \times 10)$. e Multiple millimetric hemangiomas characterized by congestive ectatic vessels are disseminated within the hepatic parenchyma $(\mathrm{HES} \times 0.6)$. 
Table 1. Twenty informative cases of giant pedunculated hepatic hemangiomas described in the literature

\begin{tabular}{|c|c|c|c|c|c|c|}
\hline Ref.; case No. & $\begin{array}{l}\text { Sex/ } \\
\text { age }\end{array}$ & Initial clinical symptoms & $\begin{array}{l}\text { Liver } \\
\text { lobe }\end{array}$ & $\begin{array}{l}\text { Pathological } \\
\text { size }^{\mathrm{a}}\end{array}$ & Radiological exams & $\begin{array}{l}\text { Treat- } \\
\text { ment }\end{array}$ \\
\hline 7; case No. 1 & $\mathrm{~F} / 53$ & Abdominal mass, pain & Right & NA & CT scan/MRI & Surgery \\
\hline 7 ; case No. 2 & $\mathrm{M} / 71$ & No & Left & NA & CT scan & Surgery \\
\hline 7; case No. 3 & $\mathrm{~F} / 47$ & Abdominal pain & Left & 8.5 & US, CT scan & Surgery \\
\hline 7; case No. 4 & $\mathrm{~F} / 60$ & Obstructive icterus & Left & 15 & US, CT scan, scintigraphy & Surgery \\
\hline 7; case No. 5 & $\mathrm{~F} / 55$ & Abdominal pain and discomfort & Left & NA & CT scan, scintigraphy & Surgery \\
\hline 7; case No. 6 & $\mathrm{~F} / 53$ & No & Left & 15 & CT scan & Surgery \\
\hline 7; case No. 7 & $\mathrm{M} / 67$ & No & Right & 11 & US, CT scan, angiography & Surgery \\
\hline 7; case No. 8 & F/NA & No & Left & NA & US, CT scan & Surgery \\
\hline 7; case No. 9 & $\mathrm{M} / 58$ & Abdominal pain & Right & 4 & US, CT scan & Surgery \\
\hline 7; case No. 10 & $\mathrm{M} / 48$ & No & Right & 4 & US, CT scan & Surgery \\
\hline 7; case No. 11 & $\mathrm{~F} / 45$ & No & Right & 4 & US, CT scan & Surgery \\
\hline 7; case No. 12 & $\mathrm{~F} / 48$ & Abdominal pain & Left & 12 & US, CT scan & Surgery \\
\hline 10 & $\mathrm{M} / 45$ & Acute "appendicitis" & Left & 13 & CT scan & Surgery \\
\hline 6 & $\mathrm{~F} / 31$ & Acute “appendicitis" (torsion) & Right & 15 & CT scan & Surgery \\
\hline 9 & $\mathrm{~F} / 56$ & Abdominal discomfort, dyspepsia & Left & 4.5 & CT scan & Surgery \\
\hline 8 & $\mathrm{~F} / 63$ & Acute intestinal obstruction & Right & 14 & CT scan, MRI & Surgery \\
\hline 3 & $\mathrm{~F} / 37$ & Abdominal pain & Left & 10 & CT scan & Surgery \\
\hline 4 & $\mathrm{~F} / 28$ & Abdominal palpable mass, discomfort & Right & 18 & US, CT scan, MRI & Surgery \\
\hline 5 & $\mathrm{~F} / 45$ & Acute abdominal pain (torsion) & Left & 10.5 & US, CT scan & Surgery \\
\hline Our case & $\mathrm{M} / 58$ & Abdominal pain, diarrhea, vomiting & Left & 20 & US, CT scan, MRI & Surgery \\
\hline
\end{tabular}

NA, not available. a Size is indicated in centimeters. 